\title{
CORRIGENDUM: NEW NUMERICAL APPROACH FOR FRACTIONAL DIFFERENTIAL EQUATIONS
}

\author{
Abdon Atangana ${ }^{1}$ And Kolade M. Owolabi ${ }^{1,2, *}$ [
}

Accepted July 1, 2021.

Correction to: [MMNP $\mathbf{1 3}$ (2018) 3]

\section{INTRODUCTION}

In this paper, we briefly provide some corrections to our previously published article in [1] as pointed out by some anonymous reviewers. The original published article can be accessed online via https://doi.org/10.1051/ $\mathrm{mmnp} / 2018010$.

\section{In the abstract}

The sentence, "The Adams-Bashforth method for fractional differentiation suggested and are commonly use in the literature nowadays is not mathematically correct and the method was derived without taking into account the nonlinearity of the power law kernel" The phrase "not mathematically correct" was intended to be "mathematically correct". Also, "without taking into account" should be "taking into account"

\section{Just below equation (3.3)}

Thus, at $t=t_{n+1}, n=0,1,2, \ldots$ should be $t=t_{n+1}, n=1$.

$$
\begin{gathered}
A_{\alpha, 2}=\int_{0}^{t_{n}}\left(t_{n}-t\right)^{\alpha-1} f(t, y(t)) \mathrm{d} t \\
A_{\alpha, 1}=\frac{f\left(t_{n}, y_{n}\right)}{h \Gamma(\alpha)}\left[\int_{0}^{t_{n+1}} t\left(t_{n+1}-t\right)^{\alpha-1} \mathrm{~d} t-\int_{0}^{t_{n+1}} t_{n-1}\left(t_{n+1}-t\right)^{\alpha-1} \mathrm{~d} t\right] \\
-\frac{f\left(t_{n-1}, y_{n-1}\right)}{h \Gamma(\alpha)}\left[\int_{0}^{t_{n+1}} t\left(t_{n+1}-t\right)^{\alpha-1} \mathrm{~d} t-\int_{0}^{t_{n+1}} t_{n}\left(t_{n+1}-t\right)^{\alpha-1} \mathrm{~d} t\right] \\
=\frac{f\left(t_{n}, y_{n}\right)}{h \Gamma(\alpha)}\left\{\frac{t_{n+1}^{\alpha+1}}{\alpha+1}-t_{n-1} t_{n+1}^{\alpha}\right\}-\frac{f\left(t_{n-1}, y_{n-1}\right)}{h \Gamma(\alpha)}\left\{\frac{t_{n+1}^{\alpha+1}}{\alpha+1}-t_{n} t_{n+1}^{\alpha}\right\} \\
A_{\alpha, 2}=\frac{f\left(t_{n}, y_{n}\right)}{h \Gamma(\alpha)}\left\{\frac{t_{n}^{\alpha}}{\alpha+1}-t_{n-1} t_{n}^{\alpha}\right\}-\frac{f\left(t_{n-1}, y_{n-1}\right)}{h \Gamma(\alpha)}\left\{\frac{t_{n}^{\alpha+1}}{\alpha+1}-t_{n}^{\alpha+1}\right\} .
\end{gathered}
$$

\footnotetext{
${ }^{1}$ Institute for Groundwater Studies, Faculty of Natural and Agricultural Sciences, University of the Free State, Bloemfontein 9300, South Africa.

2 Department of Mathematical Sciences, Federal University of Technology, PMB 704, Akure, Ondo State, Nigeria.

* Corresponing author: mkowolax@yahoo.com
} 
TABLE 1. Error report for different discretization steps with $\alpha=0.95, \beta=1$ and $t=2$.

\begin{tabular}{cc}
\hline Step-size $(h)$ & Error \\
\hline$h=1.0$ & 0.5017 \\
$h=0.1$ & 0.0070 \\
$h=0.01$ & 0.0021 \\
$h=0.001$ & 0.0020 \\
\hline
\end{tabular}

\section{Equation (3.11)}

$$
\begin{aligned}
y_{n+1}= & y_{n}+\frac{f\left(t_{n}, y_{n}\right) h^{\alpha}}{\Gamma(\alpha)}\left\{\frac{(n+1)^{\alpha+1}}{\alpha+1}-(n-1)(n+1)^{\alpha}-\frac{n^{\alpha+1}}{\alpha+1}+(n-1) n^{\alpha}\right\} \\
& -\frac{f\left(t_{n-1}, y_{n-1}\right) h^{\alpha}}{\Gamma(\alpha)}\left\{\frac{(n+1)^{\alpha+1}}{\alpha+1}-(n+1)^{\alpha} n-\frac{n^{\alpha+1}}{\alpha+1}+n^{\alpha+1}\right\}
\end{aligned}
$$

\section{Remark}

When $\alpha=1$, the above equation gives

$$
\begin{aligned}
y_{n+1}= & y_{n}+\frac{f\left(t_{n}, y_{n}\right) h}{\Gamma(1)}\left\{\frac{(n+1)^{2}}{2}-(n-1)(n+1)-\frac{n^{2}}{2}+(n-1) n\right\} \\
& +\frac{f\left(t_{n-1}, y_{n-1}\right)}{\Gamma(1)}\left\{\frac{(n+1)^{2}}{2}-(n+1) n-\frac{n^{2}}{2}+n^{2}\right\} .
\end{aligned}
$$

By simplifying further, we have

$$
y_{n+1}=y_{n}+\frac{3}{2} h f\left(t_{n}, y_{n}\right)-\frac{h}{2} f\left(t_{n-1}, y_{n-1}\right)
$$

which is indeed the classical Adams-Bashforth scheme.

\section{Test problem}

Consider the following Caputo fractional differential equation

$$
{ }_{0}^{C} \mathcal{D}_{t}^{\alpha} y(t)=t^{\beta}, \quad y(0)=0, \quad \beta>0,0<\alpha \leq 1 .
$$

We obtain the exact solution of (1.2) by applying the Caputo definition to obtain

$$
y(t)=\frac{\Gamma(\beta+1)}{\Gamma(\alpha+\beta+1)} t^{\alpha+\beta}
$$

By using above numerical scheme, we conduct the simulation experiment for $\alpha=\frac{19}{20}$ for different instances of discretization step-size $h$ to obtain results in Table 1 . The error in the estimation is defined by

$$
\left\|R_{\alpha}\right\|=\left\|y_{\text {approximate }}(t)-y_{\text {exact }}(t)\right\|_{\infty} .
$$

The comparison between the numerical and exact solutions is displayed in Figure 1 for different step-sizes and $\beta$. Parameters are given in the figure caption. 

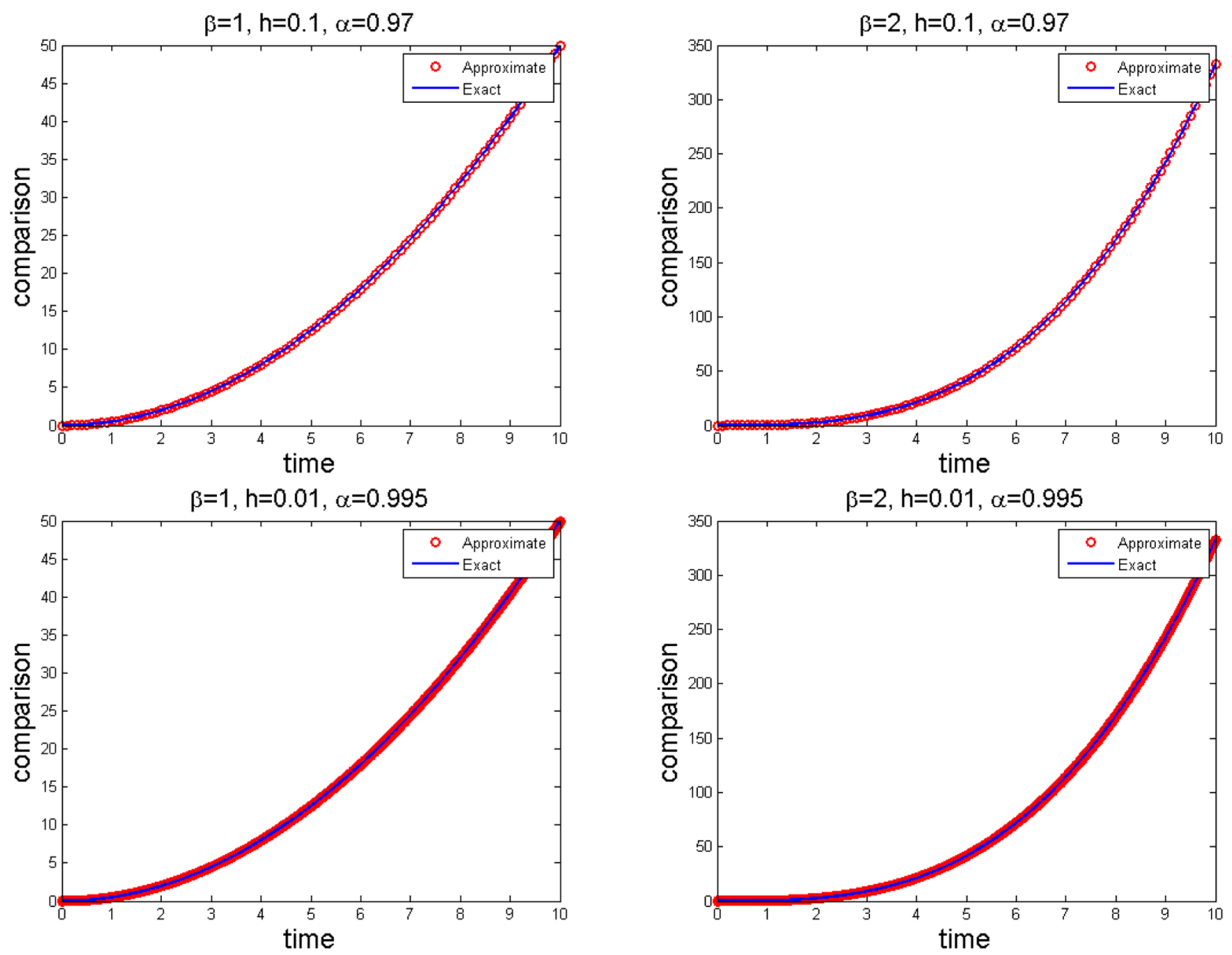

FiguRE 1. Numerical results for Caputo fractional problem (1.2) showing the comparison between the exact and approximate (numerical) results for $t=10$. Take note of the variation in the amplitudes.

We shall remark that this approach is very accurate when the fractional order is above 0.75 and $\beta \in[2,4]$ regardless of chosen simulation time $t$. Thus, for $0<\alpha<0.75$, we suggest the following

$$
\begin{aligned}
y_{n+1}= & y_{n}+\frac{1}{\Gamma(\alpha)} \sum_{j=0}^{n} \int_{t_{j}}^{t_{j+1}}\left(t_{n+1}-\tau\right)^{\alpha-1} f(\tau, y(\tau)) \mathrm{d} \tau \\
& -\frac{1}{\Gamma(\alpha)} \sum_{j=0}^{n-1} \int_{t_{j}}^{t_{j+1}}\left(t_{n}-\tau\right)^{\alpha-1} f(\tau, y(\tau)) \mathrm{d} \tau
\end{aligned}
$$

Within $\left[t_{j}, t_{j+1}\right]$

$$
f(\tau, y(\tau)) \simeq P_{j}(\tau)=\frac{\tau-t_{j-1}}{\Delta t} f\left(t_{j}, y\left(t_{j}\right)\right)-\frac{\tau-t_{j}}{\Delta t} f\left(t_{j-1}, y\left(t_{j-1}\right)\right)
$$


replacing in both yields

$$
\begin{aligned}
y_{n+1}= & y_{n}+\frac{(\Delta t)^{\alpha}}{\Gamma(\alpha+2)} \sum_{j=0}^{n} f\left(t_{j}, y\left(t_{j}\right)\right)\left[(n-j+\alpha+2)(n-j+1)^{\alpha}-(n-j)^{\alpha}(n-j+2 \alpha+2)\right] \\
& -\frac{(\Delta t)^{\alpha}}{\Gamma(\alpha+2)} \sum_{j=0}^{n} f\left(t_{j-1}, y\left(t_{j-1}\right)\right)\left[(n-j+1)^{\alpha+1}-(n-j)(n-j+1 \alpha)\right] \\
& -\frac{(\Delta t)^{\alpha}}{\Gamma(\alpha+2)} \sum_{j=1}^{n-1} f\left(t_{j}, y\left(t_{j}\right)\right)\left[(n-j+\alpha+1)(n-j)^{\alpha}-(n-j-1)^{\alpha}(n-j+2 \alpha+1)\right] \\
& +\frac{(\Delta t)^{\alpha}}{\Gamma(\alpha+2)} \sum_{j=0}^{n-1} f\left(t_{j-1}, y\left(t_{j-1}\right)\right)\left[(n-j)^{\alpha+1}-(n-j-1)^{\alpha}(n-j+\alpha)\right]
\end{aligned}
$$

Theorem 3.1 Let consider the following nonlinear fractional Cauchy problem

$$
{ }_{0}^{C} \mathcal{D}_{t}^{\alpha} y(t)=f(t, y(t))
$$

such that $f \in C^{\alpha}$ and that $\frac{\partial^{2} f}{\partial t^{2}}(t, y(t))$ is bounded. Then,

$$
\begin{aligned}
y_{n+1}= & y_{n}+\frac{f\left(t_{n}, y_{n}\right)}{h \Gamma(\alpha)}\left\{\frac{t_{n+1}^{\alpha+1}}{\alpha+1}-t_{n} t_{n+1}^{\alpha}-\frac{t_{n}^{\alpha+1}}{\alpha+1}+t_{n-1} t_{n}^{\alpha}\right\} \\
& +\frac{f\left(t_{n-1}, y_{n-1}\right)}{h \Gamma(\alpha)}\left\{\frac{t_{n+1}^{\alpha+1}}{\alpha+1}-t_{n} t_{n+1}^{\alpha}-\frac{t_{n}^{\alpha+1}}{\alpha+1}+t_{n}^{\alpha+1}\right\}+R_{n}^{\alpha}(\zeta)
\end{aligned}
$$

with $\left|R_{n}^{\alpha}(\zeta)\right|<K(\Delta t)^{\alpha+2} g(\alpha, n)$

Proof: Under the condition presented above

$$
\begin{aligned}
y_{n+1}= & y_{n}+\frac{f\left(t_{n}, y_{n}\right)}{h \Gamma(\alpha)}\left\{\frac{t_{n+1}^{\alpha+1}}{\alpha+1}-t_{n} t_{n+1}^{\alpha}-\frac{t_{n}^{\alpha+1}}{\alpha+1}+t_{n-1} t_{n}^{\alpha}\right\} \\
& +\frac{f\left(t_{n-1}, y_{n-1}\right)}{h \Gamma(\alpha)}\left\{\frac{t_{n+1}^{\alpha+1}}{\alpha+1}-t_{n} t_{n+1}^{\alpha}-\frac{t_{n}^{\alpha+1}}{\alpha+1}+t_{n}^{\alpha+1}\right\} \\
& +\frac{1}{\Gamma(\alpha)}\left\{\left.\int_{0}^{t_{n+1}}\left(t_{n+1}-\tau\right)^{\alpha-1} \frac{\left(t-t_{n}\right)\left(t-t_{n-1}\right)}{2 !} \frac{\partial^{2} f}{\partial t^{2}}(t, y(t))\right|_{t=\zeta} \mathrm{d} t\right. \\
& \left.-\left.\int_{0}^{t_{n}}\left(t_{n}-\tau\right)^{\alpha-1} \frac{\left(t-t_{n}\right)\left(t-t_{n-1}\right)}{2 !} \frac{\partial^{2} f}{\partial t^{2}}(t, y(t))\right|_{t=\zeta} \mathrm{d} t\right\} .
\end{aligned}
$$

Here,

$$
\begin{aligned}
R_{n}^{\alpha}(\zeta)= & \frac{1}{\Gamma(\alpha)}\left\{\left.\int_{0}^{t_{n+1}}\left(t_{n+1}-t\right)^{\alpha-1}\left(t-t_{n}\right)\left(t-t_{n-1}\right) \frac{\partial^{2} f}{\partial t^{2}}(t, y(t))\right|_{t=\zeta} \mathrm{d} t\right. \\
& \left.-\int_{0}^{t_{n}}\left(t_{n}-t\right)^{\alpha-1}\left(t-t_{n}\right)\left(t-t_{n-1}\right) \frac{\partial^{2} f}{\partial t^{2}}(t, y(t)) \mathrm{d} t\right\}
\end{aligned}
$$


The above can be reformulated as

$$
\begin{aligned}
R_{n}^{\alpha}(\zeta)= & \frac{1}{\Gamma(\alpha)}\left[\left.\int_{0}^{t_{n+1}}\left(t_{n+1}-\tau\right)^{\alpha-1}\left\{t^{2}-\left(t_{n}+t_{n-1}\right) t+t_{n} t_{n-1}\right\} \frac{\partial^{2} f}{\partial t^{2}}(t, y(t))\right|_{t=\zeta} \mathrm{d} t\right. \\
& \left.\int_{0}^{t_{n}}\left(t_{n}-\tau\right)^{\alpha-1}\left\{t^{2}-\left(t_{n}+t_{n-1}\right) t+t_{n} t_{n-1}\right\} \frac{\partial^{2} f}{\partial t^{2}}(t, y(t)) \mathrm{d} t\right]
\end{aligned}
$$

Thus,

$$
\begin{aligned}
\left|R_{n}^{\alpha}(\zeta)\right|< & \frac{1}{\Gamma(\alpha)}\left\{\int_{0}^{t_{n+1}} t^{2}\left(t_{n+1}-t\right)^{\alpha-1}\left|\frac{\partial^{2} f}{\partial t^{2}}(t, y(t))\right|_{t=\zeta} \mid \mathrm{d} t\right. \\
& \left.+\left.\int_{0}^{t_{n+1}} t\left(t_{n+1}-t\right)^{\alpha-1}\left|\frac{\partial^{2} f}{\partial t^{2}}(t, y(t))\right|_{t=\zeta}\left|+\int_{0}^{t_{n}} t^{2}\left(t_{n}-t\right)^{\alpha-1}\right| \frac{\partial^{2} f}{\partial t^{2}}(t, y(t))\right|_{t=\zeta} \mid\right\} \\
& <\frac{1}{\Gamma(\alpha)}\left\{\int_{0}^{t_{n+1}} t^{2}\left(t_{n+1}-t\right)^{\alpha-1} \sup _{\ell \in(0, t]}\left|\frac{\partial^{2} f}{\partial \ell^{2}}(\ell, y(\ell))\right|_{\ell=\zeta} \mid \mathrm{d} t\right. \\
& +\left(t_{n}+t_{n-1}\right) \int_{0}^{t_{n+1}} t\left(t_{n+1}-t\right)^{\alpha-1} \sup _{\ell \in(0, t]}\left|\frac{\partial^{2} f}{\partial \ell^{2}}(\ell, y(\ell))\right|_{\ell=\zeta} \mid \mathrm{d} t \\
& +\left(t_{n} t_{n-1}\right) \int_{0}^{t_{n+1}}\left(t_{n+1}-t\right)^{\alpha-1} \sup _{\ell \in(0, t]}\left|\frac{\partial^{2} f}{\partial \ell^{2}}(\ell, y(\ell))\right|_{\ell=\zeta} \mid \mathrm{d} t \\
& +\int_{0}^{t_{n}}\left(t_{n}-t\right)^{\alpha-1} t^{2} \sup _{\ell \in(0, t]}\left|\frac{\partial^{2} f}{\partial \ell^{2}}(\ell, y(\ell))\right|_{\ell=\zeta} \mid \mathrm{d} t \\
& +\int_{0}^{t_{n}}\left(t_{n}-t\right)^{\alpha-1} t\left(t_{n}+t_{n-1}\right) \sup _{\ell \in(0, t]}\left|\frac{\partial^{2} f}{\partial \ell^{2}}(\ell, y(\ell))\right|_{\ell=\zeta} \mid \mathrm{d} t \\
& \left.\int_{0}^{t_{n}} t_{n} t_{n-1}\left(t_{n}-t\right)^{\alpha-1} \sup _{\ell \in(0, t]}\left|\frac{\partial^{2} f}{\partial \ell^{2}}(\ell, y(\ell))\right|_{\ell=\zeta} \mid \mathrm{d} t\right\}
\end{aligned}
$$

Since $\frac{\partial^{2} f}{\partial t^{2}}$ is bounded, then

$$
\sup _{t \in(0, t]}\left|\frac{\partial^{2} f}{\partial t^{2}}(t, y(t))\right|_{\ell=\zeta}<K .
$$

Thus

$$
\begin{aligned}
\left|R_{n}^{\alpha}(\zeta)\right|< & K(\Delta t)^{(\alpha+2)}\left\{\frac{2(n+1)^{\alpha+2}}{\Gamma(\alpha+3)}+\frac{(2 n-1)(n+1)^{\alpha+1}}{\Gamma(\alpha+2)}+\frac{n(n-1)(n+1)^{\alpha}}{\Gamma(\alpha+1)}\right. \\
& \left.+\frac{2 n^{\alpha+2}}{\Gamma(\alpha+3)}+\frac{n^{\alpha+2}(2 n-1)}{\Gamma(\alpha+2)}+\frac{n^{\alpha+1}(n-1)}{\Gamma(\alpha+1)}\right\}
\end{aligned}
$$

\section{Correction to subsection 3.3}

$$
\begin{aligned}
A_{\alpha, 1} & =\frac{\alpha}{\Gamma(\alpha) A B(\alpha)} \int_{0}^{t_{n+1}}\left(t_{n+1}-t\right)^{\alpha-1}\left\{\frac{t-t_{n-1}}{h} f\left(t_{n}, y_{n}\right)-\frac{t-t_{n}}{h} f\left(t_{n-1}, y_{n-1}\right)\right\} \mathrm{d} \tau \\
& =\frac{f\left(t_{n}, y_{n}\right)}{\Gamma(\alpha) A B(\alpha) h}\left\{\frac{t_{n+1}^{\alpha+1}}{\alpha+1}-t_{n-1} t_{n+1}^{\alpha}\right\}-\frac{f\left(t_{n-1}, y_{n-1}\right)}{h \Gamma(\alpha) A B(\alpha)}\left\{\frac{t_{n+1}^{\alpha+1}}{\alpha+1}-t_{n} t_{n+1}^{\alpha}\right\}
\end{aligned}
$$


and

$$
A_{\alpha, 2}=\frac{f\left(t_{n}, y_{n}\right)}{h \Gamma(\alpha) A B(\alpha)}\left\{\frac{t_{n}^{\alpha+1}}{\alpha+1}-t_{n-1} t_{n}^{\alpha}\right\}-\frac{f\left(t_{n-1}, y_{n-1}\right)}{h \Gamma(\alpha) A B(\alpha)}\left\{\frac{t_{n}^{\alpha+1}}{\alpha+1}-t_{n}^{\alpha+1}\right\} .
$$

Equation (3.32) should be

$$
\begin{aligned}
y\left(t_{n+1}\right)-y\left(t_{n}\right) & =\frac{1-\alpha}{A B(\alpha)}\left\{f\left(t_{n}, y_{n}\right)-f\left(t_{n+1}, y_{n-1}\right)\right\}+\frac{f\left(t_{n}, y_{n}\right)}{h \Gamma(\alpha) A B C(\alpha)}\left\{\frac{t_{n+1}^{\alpha+1}}{\alpha+1}-t_{n} t_{n+1}^{\alpha}\right\} \\
& -\frac{f\left(t_{n-1}, y_{n-1}\right)}{h \Gamma(\alpha) A B C(\alpha)}\left\{\frac{t_{n+1}^{\alpha+1}}{\alpha+1}-t_{n} t_{n+1}^{\alpha}\right\}-\frac{f\left(t_{n}, y_{n}\right)}{h \Gamma(\alpha) A B C(\alpha)}\left\{\frac{t_{n}^{\alpha+1}}{\alpha+1}-t_{n-1} t_{n}^{\alpha}\right\} \\
& +\frac{f\left(t_{n-1}, y_{n-1}\right)}{h \Gamma(\alpha) A B C(\alpha)}\left\{\frac{t_{n}^{\alpha+1}}{\alpha+1}-t_{n}^{\alpha+1}\right\} .
\end{aligned}
$$

Therefore,

$$
\begin{aligned}
y_{n+1}= & y_{n}+\frac{1-\alpha}{A B(\alpha)}\left\{f\left(t_{n}, y_{n}\right)-f\left(t_{n-1}, y_{n-1}\right)\right\} \\
& +\frac{f\left(t_{n}, y_{n}\right) h^{\alpha}}{A B(\alpha) \Gamma(\alpha)}\left\{\frac{(n+1)^{\alpha+1}}{\alpha+1}-(n-1)(n+1)^{\alpha}-\frac{n^{\alpha+1}}{\alpha+1}+(n-1) n^{\alpha}\right\} \\
& -\frac{f\left(t_{n-1}, y_{n-1}\right) h^{\alpha}}{A B(\alpha) \Gamma(\alpha)}\left\{\frac{(n+1)^{\alpha+1}}{\alpha+1}-n(n+1)^{\alpha}-\frac{n^{\alpha+1}}{\alpha+1}+n^{\alpha+1}\right\} .
\end{aligned}
$$

\section{Remark}

In the Atangana-Baleanu case, when $\alpha=1$, we have

$$
y_{n+1}=y_{n}+\frac{3}{2} h f\left(t_{n}, y_{n}\right)-\frac{h}{2} f\left(t_{n-1}, y_{n-1}\right)
$$

In Theorem 3.4,

$$
\begin{aligned}
R_{\alpha}= & \frac{\alpha}{A B(\alpha) \Gamma(\alpha)}\left\{\left.\int_{0}^{t_{n+1}}\left(\tau-t_{n}\right)\left(\tau-t_{n-1}\right)\left(t_{n+1}-\tau\right)^{\alpha-1} \frac{\partial^{2} f}{\partial \tau^{2}}(\tau, y(\tau))\right|_{\tau=\zeta} \mathrm{d} \tau\right. \\
& \left.-\left.\int_{0}^{t}\left(\tau-t_{n}\right)\left(\tau-t_{n-1}\right)\left(t_{n}-\tau\right)^{\alpha-1} \frac{\partial^{2} f}{\partial \tau^{2}}(\tau, y(\tau))\right|_{\tau=\zeta} \mathrm{d} \tau\right\},
\end{aligned}
$$

which implies that

$$
\begin{aligned}
R_{\alpha}= & \frac{\alpha}{A B(\alpha) \Gamma(\alpha)}\left\{\left.\int_{0}^{t_{n+1}}\left(\tau^{2}-\left(t_{n}+t_{n-1}\right) \tau+t_{n-1} t_{n}\right)\left(t_{n+1}-\tau\right)^{\tau-1} \frac{\partial^{2} f}{\partial \tau^{2}}(\tau, y(\tau))\right|_{\tau=\zeta} \mathrm{d} \tau\right. \\
& \left.-\left.\int_{0}^{t}\left(\tau^{2}-\left(t_{n}+t_{n-1}\right) \tau+t_{n-1} t_{n}\right)\left(t_{n}-\tau\right)^{\alpha-1} \frac{\partial^{2} f}{\partial \tau^{2}}(\tau, y(\tau))\right|_{\tau=\zeta} \mathrm{d} \tau\right\} .
\end{aligned}
$$


CORRIGENDUM: NEW NUMERICAL APPROACH FOR FRACTIONAL DIFFERENTIAL EQUATIONS

7
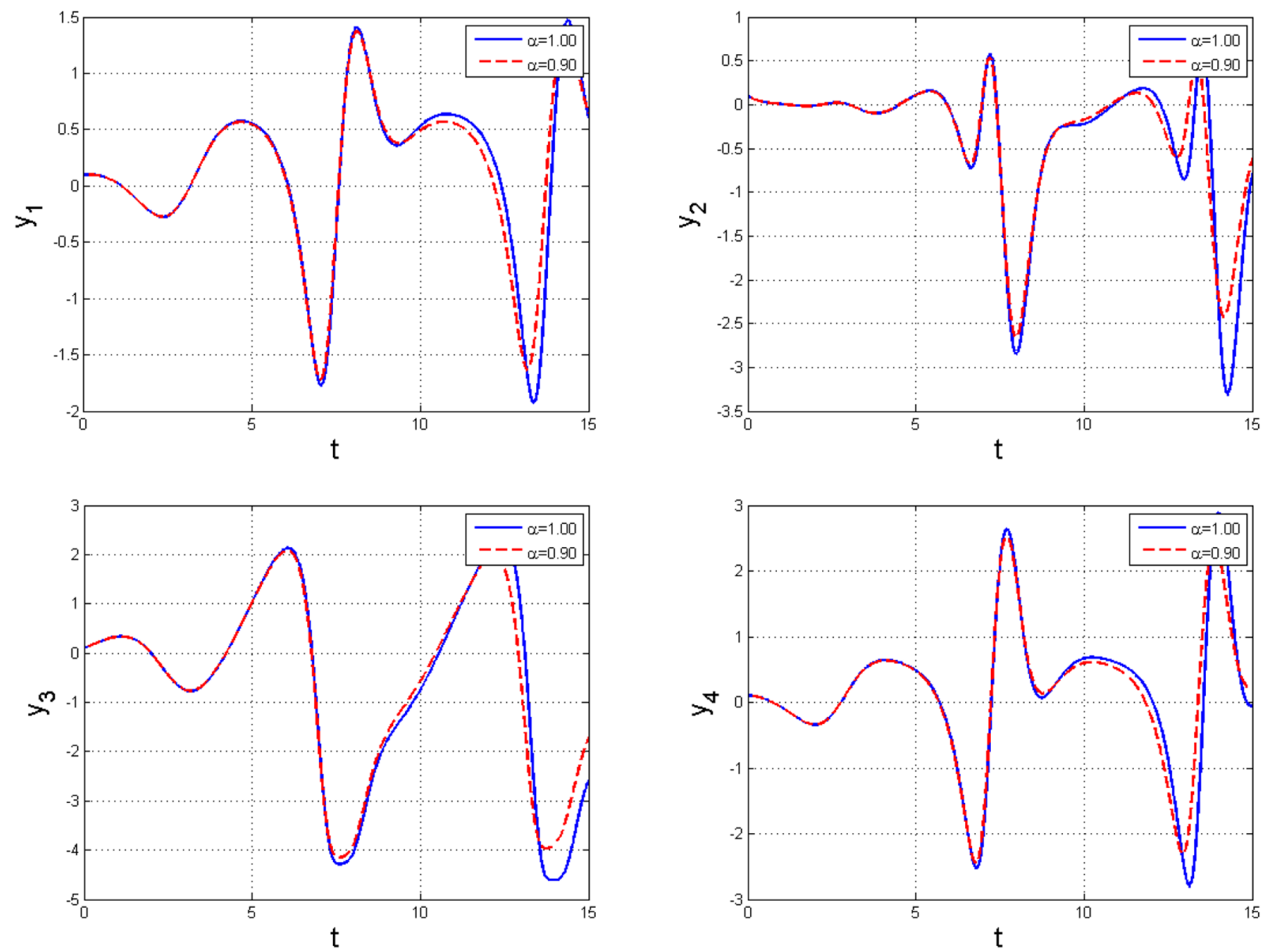

Figure 2. Time distribution of system (1.4) showing comparison between the classical and fractional order cases.

Thus,

$$
\begin{aligned}
\left|R_{\alpha}\right|< & \frac{\alpha}{A B(\alpha) \Gamma(\alpha)}\left\{\int_{0}^{t_{n+1}} \tau^{2}\left(t_{n+1}-\tau\right)^{\tau-1}\left|\frac{\partial^{2} f}{\partial \tau^{2}}(\tau, y(\tau))\right|_{\tau=\zeta} \mid \mathrm{d} \tau\right. \\
& +\left(t_{n+1}+t_{n}\right) \int_{0}^{t_{n+1}} \tau\left(t_{n+1}-\tau\right)^{\tau-1}\left|\frac{\partial^{2} f}{\partial \tau^{2}}(\tau, y(\tau))\right|_{\tau=\zeta} \mid \mathrm{d} \tau \\
& +t_{n+1} \cdot t_{n} \int_{0}^{t_{n+1}}\left(t_{n+1}-\tau\right)^{\alpha-1}\left|\frac{\partial^{2} f}{\partial \tau^{2}}(\tau, y(\tau))\right|_{\tau=\zeta} \mid \mathrm{d} \tau \\
& +\int_{0}^{t_{n}}\left(t_{n}-\tau\right)^{\alpha-1} \tau^{2}\left|\frac{\partial^{2} f}{\partial \tau^{2}}(\tau, y(\tau))\right|_{\tau=\zeta} \mid \mathrm{d} \tau \\
& +\left(t_{n-1}+t_{n}\right) \int_{0}^{t_{n}}\left(t_{n}-\tau\right)^{\alpha-1} \tau\left|\frac{\partial^{2} f}{\partial \tau^{2}}(\tau, y(\tau))\right|_{\tau=\zeta} \mid \mathrm{d} \tau \\
& \left.+t_{n} t_{n-1} \int_{0}^{t_{n}}\left(t_{n}-\tau\right)^{\alpha-1}\left|\frac{\partial^{2} f}{\partial \tau^{2}}(\tau, y(\tau))\right|_{\tau=\zeta} \mid \mathrm{d} \tau\right\} .
\end{aligned}
$$



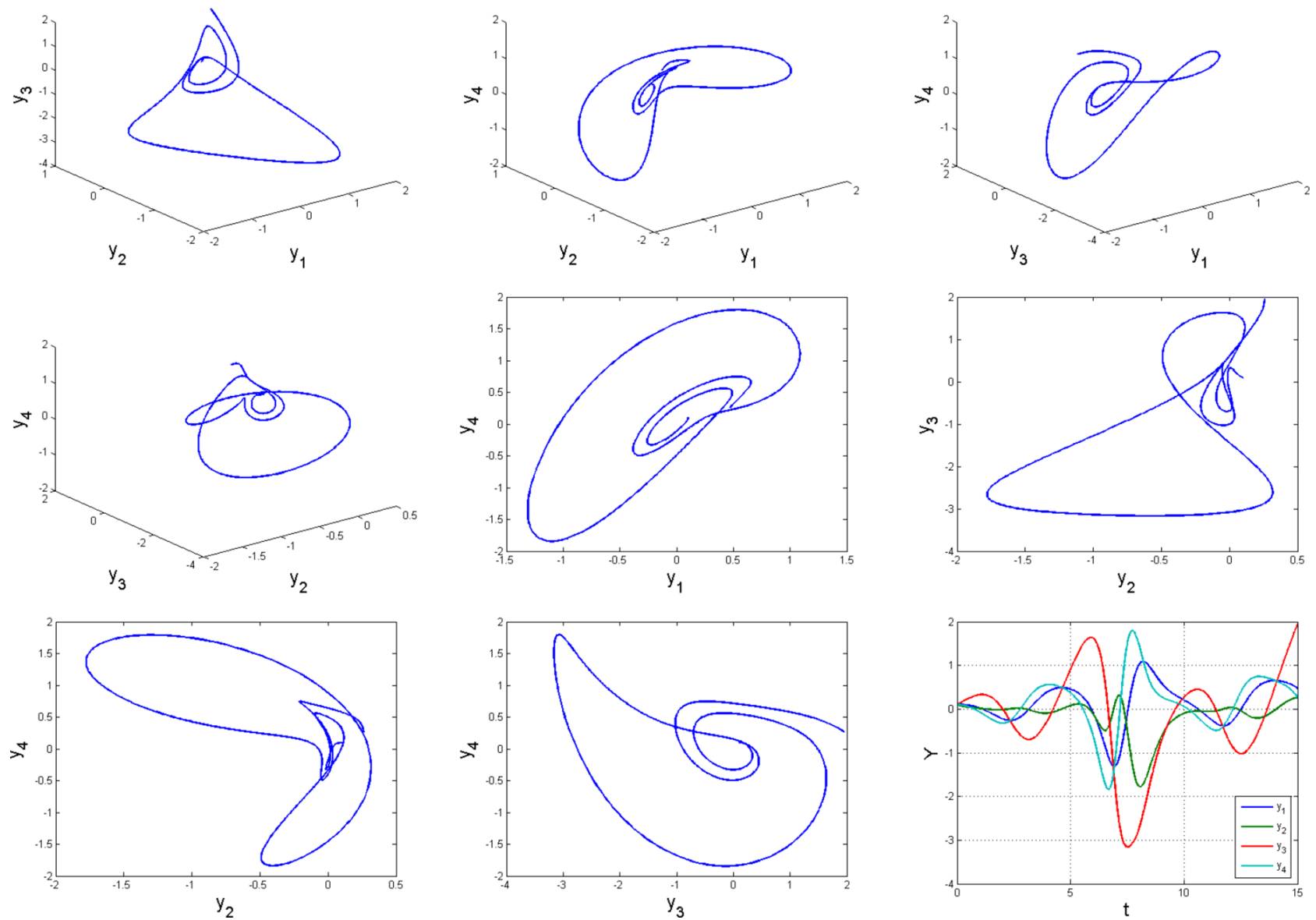

Figure 3. Evolution of chaotic system (1.4) in 2D and 3D for $\alpha=0.88$. Simulation runs for time $t=15$.

Since we assume that $\frac{\partial^{2} f}{\partial \tau^{2}}$ is bounded, then

$$
\begin{gathered}
\left|R_{\alpha}\right|<\frac{\alpha}{A B(\alpha) \Gamma(\alpha)} \sup _{t \in[0, T]}\left|\frac{\partial^{2} f}{\partial t^{2}}(t, y(t))\right|_{t=\zeta} \mid\left\{\frac{t_{n+1}^{\alpha+2} \Gamma(3) \Gamma(\alpha)}{\Gamma(\alpha+3)}+\frac{t_{n+1}^{\alpha+1} \Gamma(2) \Gamma(\alpha)}{\Gamma(\alpha+2)}\left(t_{n-1}+t_{n}\right)\right. \\
\left.+\frac{t_{n} t_{n-1} t_{n+1}^{\alpha} \Gamma(\alpha)}{\Gamma(\alpha+1)}+\frac{t_{n}^{\alpha+2} \Gamma(3) \Gamma(\alpha)}{\Gamma(\alpha+3)}+\frac{t_{n}^{\alpha+1} \Gamma(2) \Gamma(\alpha)}{\Gamma(\alpha+2)}\left(t_{n-1}+t_{n}\right)+\frac{t_{n}^{\alpha+1} t_{n-1} \Gamma(\alpha)}{\Gamma(\alpha+1)}\right\} \\
\left|R_{\alpha}\right|<\frac{\alpha}{A B(\alpha)}(\Delta t)^{\alpha+2} \sup _{t \in D}\left|\frac{\partial^{2} f}{\partial t^{2}}(t, y(t))\right|_{t=\zeta} \mid\left\{\frac{2(n+1)^{\alpha+2}}{\Gamma(\alpha+3)}+\frac{(2 n-1)(n+1)^{\alpha+1}}{\Gamma(\alpha+2)}\right. \\
\left.+\frac{n(n-1)(n+1)^{\alpha}}{\Gamma(\alpha+1)}+\frac{2 n^{\alpha+2}}{\Gamma(\alpha+3)}+\frac{n^{\alpha+2}(2 n-1)}{\Gamma(\alpha+2)}+\frac{n^{\alpha+1}(n-1)}{\Gamma(\alpha+1)}\right\} \\
\left|R_{\alpha}\right|<\frac{\alpha(\Delta t)^{\alpha+2}}{A B(\alpha)} \mathcal{K} \Phi(n, \alpha)
\end{gathered}
$$



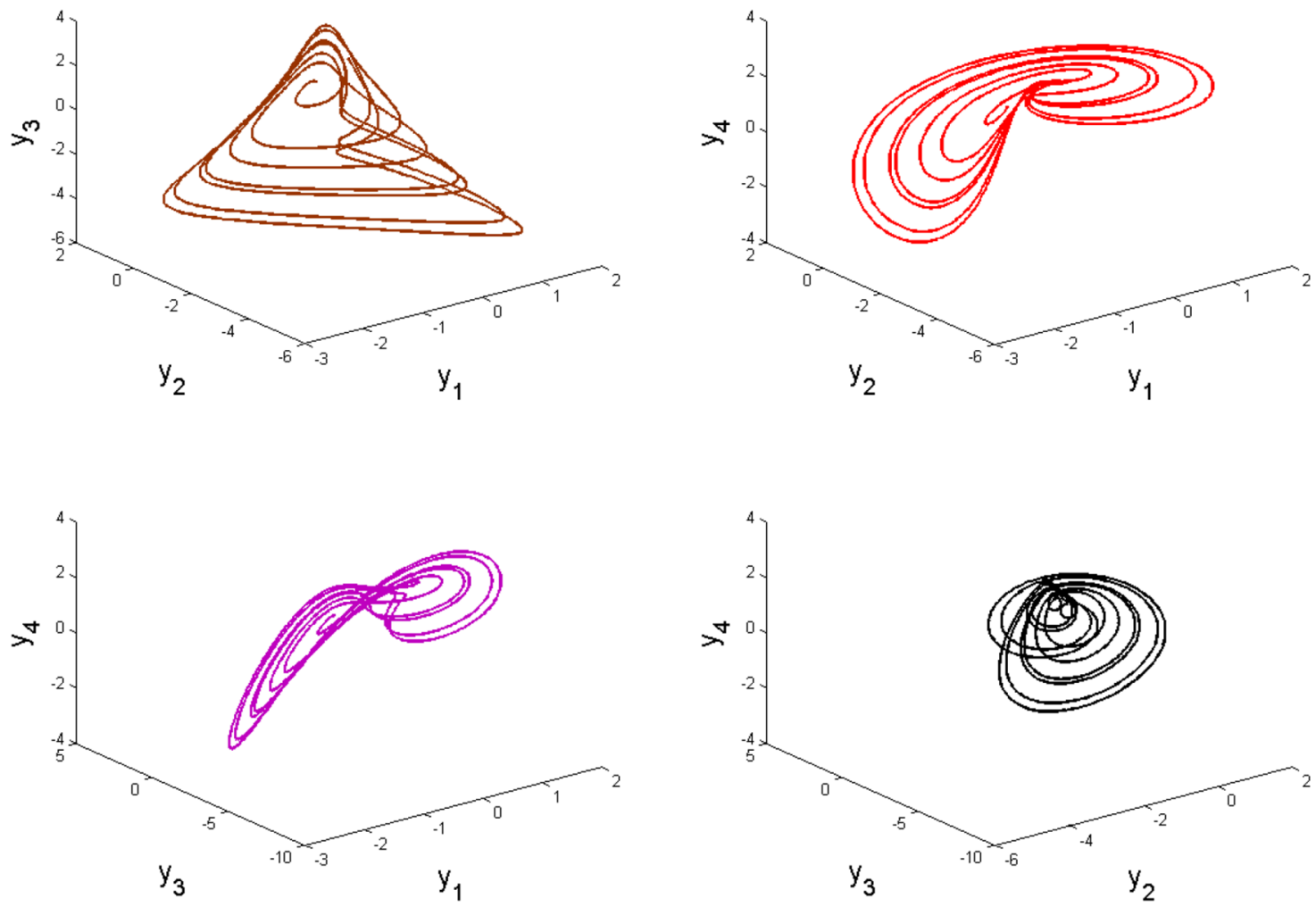

FiguRE 4. 3D evolution of system (1.4) showing chaotic oscillations for $\alpha=0.95$ with $t=40$.

where $D$ is the domain of $\frac{\partial^{2} f}{\partial t^{2}}$,

$$
\begin{aligned}
& \Phi(n, \alpha)= \frac{2(n+1)^{\alpha+2}}{\Gamma(\alpha+3)}+\frac{(2 n-1)(n+1)^{\alpha+1}}{\Gamma(\alpha+2)}+\frac{n(n-1)(n+1)^{\alpha}}{\Gamma(\alpha+1)}+\frac{2 n^{\alpha+2}}{\Gamma(\alpha+3)} \\
&+\frac{n^{\alpha+2}(2 n-1)}{\Gamma(\alpha+2)}+\frac{n^{\alpha+1}(n-1)}{\Gamma(\alpha+1)} \\
& \sup _{t \in D}\left|\frac{\partial^{2} f}{\partial t^{2}}(t, y(t))\right|_{t=\zeta} \mid<\mathcal{K} .
\end{aligned}
$$

In what follows, we test the applicability and suitability of the above numerical scheme by considering a chaotic system of coupled differential equations [2] defined by the Atangana-Baleanu fractional-order operator as

$$
\begin{aligned}
& { }_{0}^{A B C} \mathcal{D}_{t}^{\alpha} y_{1}(t)=\phi y_{4}(t)-\phi y_{1}(t), \\
& { }_{0}^{A B C} \mathcal{D}_{t}^{\alpha} y_{2}(t)=y_{3}(t) y_{4}(t)-\varphi y_{2}(t), \\
& { }_{0}^{A B C} \mathcal{D}_{t}^{\alpha} y_{3}(t)=\psi y_{1}(t)-y_{1}(t) y_{4}(t), \\
& { }_{0}^{A B C} \mathcal{D}_{t}^{\alpha} y_{4}(t)=\sigma y_{2}(t)-y_{3}(t)+y_{1}(t) y_{3}(t),
\end{aligned}
$$

where $y_{j}$ for $j=1,2,3,4$ denote the state variables with positive parameters $\phi, \varphi, \psi$ and $\sigma$. In the simulation experiment, we utilize the parameters $\phi=2, \varphi=2, \psi=3, \sigma=1$ and allow $\alpha$ to vary. The results obtained are shown in Figures 2-4. 
Acknowledgements. The authors most appreciate the Editor-in-Chief and the anonymous reviewers who spent their valuable time to read through the work and provided us with helpful comments.

\section{REFERENCES}

[1] A. Atangana and K.M. Owolabi, New numerical approach for fractional differential equations. MMNP 13 (2018) 3.

[2] C. Zhou, C. Yang, D. Xu and C. Chen, Coexisting attractors, circuit realization and impulsive synchronization of a new four-dimensional chaotic system. Mod. Phys. Lett. B 1950026 (2019) 1-16 . 